\title{
Patients' Preferences for Primary Colorectal Cancer Screening: A Survey of the National Colorectal Cancer Screening Program in Korea
}

Young-Hak $\mathrm{Cho}^{1}$, Dae Ho Kim ${ }^{1}$, Jae Myung Cha ${ }^{1,2}$, Yoon Tae Jeen ${ }^{3}$, Jeong Seop Moon ${ }^{4}$, Jin-Oh Kim ${ }^{5}$, Sang Kil Lee ${ }^{6}$, Yu Kyung $\mathrm{Cho}^{7}$, Jong Pil $\mathrm{Im}^{8}$, Jae Young Jang ${ }^{2}$, Jeong Eun Shin ${ }^{9}$, Soon Man Yoon ${ }^{10}$, Yunho Jung ${ }^{5}$, Eun Sun $\mathrm{Kim}^{3}$, Kang Nyeong Lee $^{11}$, Soo-Jeong Cho ${ }^{12}$, Yeol Kim ${ }^{13}$, and Bo Young Park ${ }^{13}$

${ }^{\prime}$ Department of Internal Medicine, Kyung Hee University Hospital at Gangdong, ${ }^{2}$ Department of Medicine, Kyung Hee University College of Medicine, ${ }^{3}$ Department of Medicine, Korea University College of Medicine, ${ }^{4}$ Department of Medicine, Inje University College of Medicine, ${ }^{5}$ Department of Medicine, Soonchunhyang University College of Medicine, ${ }^{6}$ Department of Medicine, Yonsei University College of Medicine, ${ }^{7}$ Department of Medicine, The Catholic University of Korea College of Medicine, ${ }^{8}$ Department of Medicine, Seoul National University Hospital, Seoul, ${ }^{9}$ Department of Medicine, Dankook University College of Medicine, Cheonan, ${ }^{10}$ Department of Medicine, Chungbuk National University College of Medicine, Cheongju, ${ }^{11}$ Department of Medicine, Hanyang University College of Medicine, Seoul, ${ }^{12}$ Center for Gastric Cancer, and ${ }^{13}$ Cancer Early Detection Branch, National Cancer Center, Goyang, Korea

Background/Aims: The adoption of colonoscopy as a primary colorectal cancer (CRC) screening technique has been argued for in Korea, without evidence of patient preferences. This study aimed to investigate patients' preferences for the primary CRC screening test for the National Cancer Screening Program (NCSP). Methods: Between June and August 2016, 414 individuals aged $\geq 50$ years who participated in the NCSP were prospectively invited to complete a questionnaire regarding their preferences for the primary CRC screening test and the reasons for their selection. Results: Among the 396 respondents who completed the questionnaire, 124 individuals (31.3\%) preferred the fecal immunochemical test (FIT), whereas 272 individuals (68.7\%) preferred colonoscopy. Elderly participants preferred the FIT $(p<0.001)$, whereas participants with a higher education level $(p=0.030)$, a higher income level $(p=0.009)$, or individuals with a family member $(p=0.028)$ or acquaintance $(p=0.013)$ with a history of CRC preferred colonoscopy. Only $12.9 \%$ of participants had a bad experience with a previous FIT; however, $39.3 \%$ of participants had a bad experience with a previous colonoscopy. Conclusions: Colonoscopy was preferred to FIT in a 2.2:1 ratio as the primary CRC screening test for the NCSP. Patients' preference for colonoscopy should be considered for the NCSP in Korea. (Gut Liver 2017;11:821827)

Key Words: Colorectal neoplasms; Fecal occult blood test;
Colonoscopy; Screening; Preference

\section{INTRODUCTION}

The incidence of colorectal cancer (CRC) has increased rapidly in Western and Asian countries. ${ }^{1}$ Many countries have started CRC screening programs; however, screening modalities and strategies differ among countries. ${ }^{2-4}$ In Korea, the National Cancer Screening Program (NCSP) provides a single annual fecal immunochemical test (FIT) for adults aged $\geq 50$ years, and secondary confirmatory colonoscopy for those with positive FIT results. ${ }^{5,6}$ The efficacy of a FIT-based screening program may ultimately depend on FIT and colonoscopy participation rates. However, the CRC screening rate in Korea ${ }^{7}$ has remained lower than those for other cancers, similar to other countries. ${ }^{8,9}$ Several Western countries such as Australia, Germany, Poland, Switzerland, and the United States use colonoscopy as a primary CRC screening test. ${ }^{7}$ Therefore, the adoption of colonoscopy as a primary CRC screening method has been argued in Korea, but without evidence of patient preferences.

Information about CRC screening preferences will be used to plan a primary colonoscopy screening strategy as well as to identify deficits in the current colonoscopic resources in the NCSP. However, some studies have reported variations in patient preferences for FIT $^{10-14}$ and colonoscopy ${ }^{12-18}$ as a primary CRC screening test. Information about CRC screening preference is essential for checking the availability of national resources to

Correspondence to: Jae Myung Cha

Division of Gastroenterology, Department of Internal Medicine, Kyung Hee University Hospital at Gangdong, 892 Dongnam-ro, Gangdong-gu, Seoul 05278, Korea

Tel: +82-2-440-6113, Fax: +82-2-440-6295, E-mail: drcha@khu.ac.kr

Received on January 9, 2017. Revised on February 24, 2017. Accepted on February 24, 2017. Published online July 28, 2017

pISSN 1976-2283 eISSN 2005-1212 https://doi.org/10.5009/gnl17025

() This is an Open Access article distributed under the terms of the Creative Commons Attribution Non-Commercial License (http://creativecommons.org/licenses/by-nc/4.0) which permits unrestricted non-commercial use, distribution, and reproduction in any medium, provided the original work is properly cited. 
deliver a primary screening program. Until now, however, no data have been available about patient CRC screening method preferences under the NCSP in Korea. Here we conducted a survey to assess patient preferences about CRC screening methods in the NCSP.

\section{MATERIALS AND METHODS}

\section{Patients}

Between June and August 2016, 1,093 asymptomatic subjects aged $\geq 50$ years, who participated in the NCSP, were invited to complete a questionnaire. The survey items were designed to investigate the patient's preferences regarding FIT versus colonoscopy as a primary CRC screening method. The questionnaire included questions about sex, age, education, marital status, religion, occupation, income level, drinking, smoking, comorbid disease, history of colectomy, family history of CRC in $\geq$ first-degree relatives at any age, knowledge of CRC warning symptoms, previous participation in the NCSP, previous experience with FIT or colonoscopy, and preference as well as reason for the preference of FIT or colonoscopy as the primary CRC screening test. Comorbid disease included hypertension, type II diabetes mellitus, cardiovascular disease, chronic renal disease, cerebrovascular disease or any malignancy. Respondents who did not complete the questionnaire were excluded from the analysis. All responses were anonymous, and all respondents voluntary participated. This study was approved by the Institu- tional Review Board of Kyung Hee University Hospital at Gangdong (KHNMC IRB number: 2016-05-027).

\section{Questionnaires for preference}

The questionnaire regarding primary CRC screening test preference was designed to elicit the patient's preference for the primary CRC screening method and the reasons therein. Patients were asked which screening strategy they would prefer, if given a choice in the future (Supplementary Material 1). The questionnaire asked patients about (1) experience with and opinions about the NCSP; and (2) preference and reason for the preference of a particular primary CRC screening test. The questionnaire was based on a literature review ${ }^{19-21}$ and semi-structured discussions with the "Committee of Endoscopy Quality Improvement in the Korean Society of Gastrointestinal Endoscopy.” To help participants choose an appropriate primary CRC screening test, an information sheet was provided to participants with reliable, accurate, and unbiased information about the advantages and disadvantages of FIT versus colonoscopy to enable patients to answer the study questionnaire as accurately and reliably as possible. The questionnaire was confirmed as reliable and valid by four gastroenterology fellows who were not involved in this study, and answers from this group were used to revise the survey questionnaire. Reported completion times were 10 to 15 minutes. The questions were dichotomous (yes/no) or multiple choice.

Table 1. Respondents' Demographic Characteristics and Baseline Clinical Information According to the Preferred CRC Screening Method Preference

\begin{tabular}{|c|c|c|c|}
\hline Clinical data & Preference for FIT $(n=124)$ & Preference for colonoscopy $(n=272)$ & p-value \\
\hline Age, yr & $66.17 \pm 8.09$ & $63.17 \pm 7.25$ & 0.208 \\
\hline Male sex & 39 (31.5) & $100(36.8)$ & 0.304 \\
\hline Past/current smoker & $14(11.4)$ & $42(15.5)$ & 0.278 \\
\hline Alcohol drinking & $19(15.4)$ & $70(25.8)$ & 0.022 \\
\hline Education (university or above) & $29(23.8)$ & $91(34.9)$ & 0.029 \\
\hline Marital status (married) & $113(91.1)$ & $252(93.3)$ & 0.436 \\
\hline Religion (yes) & $84(67.7)$ & $175(64.3)$ & 0.509 \\
\hline Employment & & & 0.139 \\
\hline Full-/part-time & $33(26.6)$ & $92(34.1)$ & \\
\hline Not working/homemaker & $91(73.4)$ & $178(65.9)$ & \\
\hline Income (dollars/mo) & & & 0.013 \\
\hline$<1,818$ & $75(65.2)$ & $141(54.2)$ & \\
\hline $1,818-4,545$ & $37(32.2)$ & $90(34.6)$ & \\
\hline$>4,545$ & $3(2.6)$ & $29(11.2)$ & \\
\hline Comorbid disease (yes) & $63(50.8)$ & $126(46.3)$ & 0.408 \\
\hline Previous colectomy (yes) & $2(1.6)$ & $17(6.3)$ & 0.043 \\
\hline Family history of CRC & $6(4.8)$ & $33(12.2)$ & 0.023 \\
\hline Acquaintances with CRC (yes) & $18(14.6)$ & $71(26.1)$ & 0.012 \\
\hline
\end{tabular}

Data are presented as mean \pm SD or number (\%).

CRC, colorectal cancer; FIT, fecal immunochemical test. 
Table 2. Factors Influencing Patients' Preference for a CRC Screening Method

\begin{tabular}{|c|c|c|c|}
\hline \multirow{2}{*}{ Variable } & \multicolumn{3}{|c|}{ OR $(95 \% \mathrm{CI})$} \\
\hline & FIT preference & Colonoscopy preference & $\mathrm{p}$-value \\
\hline Age group, yr & & & 0.001 \\
\hline $50-59$ & Reference & Reference & \\
\hline $60-69$ & $1.582(0.926-2.703)$ & $0.632(0.370-1.080)$ & 0.094 \\
\hline$\geq 70$ & $3.037(1.671-5.519)$ & $0.329(0.181-0.598)$ & $<0.001$ \\
\hline \multicolumn{4}{|l|}{ Sex } \\
\hline Male & Reference & Reference & \\
\hline Female & $1.267(0.806-1.992)$ & $0.789(0.502-1.240)$ & 0.305 \\
\hline \multicolumn{4}{|l|}{ Smoker } \\
\hline No & Reference & Reference & \\
\hline Yes & $0.700(0.367-1.337)$ & $1.428(0.748-2.726)$ & 0.280 \\
\hline \multicolumn{4}{|l|}{ Alcohol drinking } \\
\hline No & Reference & Reference & \\
\hline Yes & $0.525(0.300-0.918)$ & $1.906(1.089-3.336)$ & 0.024 \\
\hline \multicolumn{4}{|l|}{ Education } \\
\hline High school or less & Reference & Reference & \\
\hline University or more & $0.583(0.357-0.949)$ & $1.717(1.053-2.798)$ & 0.030 \\
\hline \multicolumn{4}{|l|}{ Marital status } \\
\hline Married & Reference & Reference & \\
\hline Unmarried & $0.734(0.336-1.604)$ & $1.363(0.623-2.980)$ & 0.438 \\
\hline \multicolumn{4}{|l|}{ Religion } \\
\hline No & Reference & Reference & \\
\hline Yes & $1.164(0.742-1.827)$ & $0.859(0.547-1.349)$ & 0.509 \\
\hline \multicolumn{4}{|c|}{ Full-/part-time employment } \\
\hline No & Reference & Reference & \\
\hline Yes & $1.425(0.890-2.283)$ & $0.702(0.438-1.124)$ & 0.140 \\
\hline Income (dollars/mo) & & & 0.025 \\
\hline$<1,818$ & Reference & Reference & \\
\hline $1,818-4,545$ & $0.773(0.481-1.242)$ & $1.294(0.805-2.079)$ & 0.287 \\
\hline$>4,545$ & $0.194(0.057-0.660)$ & $5.142(1.516-17.438)$ & 0.009 \\
\hline \multicolumn{4}{|l|}{ Comorbid disease } \\
\hline No & Reference & Reference & \\
\hline Yes & $1.197(0.782-1.831)$ & $0.836(0.546-1.278)$ & 0.408 \\
\hline \multicolumn{4}{|l|}{ Previous colectomy } \\
\hline No & Reference & Reference & \\
\hline Yes & $0.243(0.055-1.069)$ & 4.115 (0.936-18.096) & 0.061 \\
\hline \multicolumn{4}{|l|}{ Family history of CRC } \\
\hline No & Reference & Reference & \\
\hline Yes & $0.367(0.149-0.900)$ & $2.727(1.112-6.690)$ & 0.028 \\
\hline \multicolumn{4}{|l|}{ Acquaintances with CRC } \\
\hline No & Reference & Reference & \\
\hline Yes & $0.485(0.275-0.857)$ & 2.061 (1.167-3.638) & 0.013 \\
\hline
\end{tabular}

CRC, colorectal cancer; OR, odds ratio; CI, confidence interval; FIT, fecal immunochemical test. 


\section{Statistical analysis}

For the sample size estimation, we assumed that the 2-fold difference in the preference for CRC screening tools between colonoscopy and FIT would be sufficient for clinicians to recommend colonoscopy rather than FIT as a primary screening test. Based on these assumptions and 26.6\% FIT participation rate of NCSP, 2014 in Korea, a minimum of 51 subjects were required for a power of $80 \%$ to detect a difference at $\mathrm{p}<0.05$ level of significance.

Categorical variables are expressed as numbers (percentage), whereas continuous variables are expressed as mean \pm standard deviation. Factors influencing preference for CRC screening tests were evaluated using binary logistic regression with odds ratios and a 95\% confidence interval. Two-tailed p-values $<0.05$ were considered statistically significant. Statistical analyses were performed using the SPSS version 18.0 for Windows (SPSS Inc., Chicago, IL, USA).

\section{RESULTS}

Among 1,093 asymptomatic subjects aged $\geq 50$ years who participated in the NCSP in our hospital, 414 answered this survey for a response rate of 37.9\%. Overall, the survey results from 396 respondents were analyzed after the exclusion of 16 surveys because of incomplete answers $(n=4)$, duplicate answers $(n=2)$, participant age $<50(n=4)$, or no preferred primary screening $(n=8)$. The complete overall response rate was $36.2 \%$.

\section{Characteristics of the survey respondents}

The respondents included 139 men (35.1\%) and 257 women $(64.9 \%)$ with a mean age of $64.1 \pm 7.6$ years. Table 1 shows the demographic characteristics and baseline clinical information of the respondents by CRC screening method preference: FIT

Table 3. Reasons Why Patients Prefer the FIT versus Colonoscopy

\begin{tabular}{lc}
\hline \multicolumn{1}{c}{ Reason for choosing } & Percent \\
\hline FIT & 72.8 \\
FIT is convenient and simple & 2.4 \\
FIT is safe & 4.8 \\
FIT is inexpensive & 12.8 \\
Colonoscopy is too complicated a process & 7.2 \\
Other & \\
Colonoscopy & 79.4 \\
Colonoscopy is accurate & 15.8 \\
Colonoscopy can provide therapeutic options & 1.5 \\
FIT is inconvenient & 1.5 \\
Colonoscopy is convenient & 1.8 \\
Other
\end{tabular}

FIT, fecal immunochemical test. preference group versus colonoscopy preference group. In this study, colonoscopy was preferred to FIT at a ratio of 2.2 to 1 (68.7\% vs $31.3 \%$ ) as a primary CRC screening test in the NCSP. There was no intergroup difference in age or sex distribution. However, alcohol drinkers as well as participants with a higher education or income level, a previous colectomy history, a family history of CRC, or acquaintances with CRC preferred colonoscopy.

\section{Factors influencing screening method preference and reasons}

Elderly participants significantly preferred the FIT $(\mathrm{p}<0.001)$ with an age-dependent correlation (Table 2). However, sex did not influence preference. In contrast, participants who were alcohol drinkers $(p=0.024)$, had a higher education $(p=0.030)$ or income level ( $p=0.025)$, had a family history of CRC $(p=0.028)$, or had acquaintances with CRC $(p=0.013)$ preferred colonoscopy. For the patients' preferences in Table 3, FIT was preferred for its convenience and simplicity (72.8\%) as well as low cost (4.8\%). In contrast, colonoscopy was preferred for its accuracy (79.4\%) and ability to provide therapeutic options (15.8\%).

\section{Experience with and opinions about the NCSP}

More than $90 \%$ of participants frequently participated in the NCSP (Table 4). Only 12.9\% of participants had a bad experience with FIT, the major reasons for which were stool sampling (77.8\%), and storage and transportation (20.0\%). Conversely, $39.3 \%$ of participants had a bad experience with colonoscopy, primarily due to bowel preparation (68.9\%) and the complicated examination process itself (25.5\%).

\section{DISCUSSION}

Primary colonoscopy screening may be superior considering its ability to remove the adenomatous polyp, ${ }^{22}$ although FIT is a more efficient CRC screening method. ${ }^{4,23}$ Choices for CRC screening options differ among countries. ${ }^{2-4}$ Although some Western countries provide primary colonoscopy screening, ${ }^{7}$ its adoption should be based on patient preference about screening methods, socioeconomic considerations, the current capacity to perform colonoscopy in each country, and its efficacy at preventing CRC. The planning of a primary colonoscopy screening strategy in Korea should be based on NCSP participant preference data. Our results indicate that colonoscopy was preferred to FIT at a ratio of 2.2 to 1 (68.7\% vs $31.3 \%)$ as a primary CRC screening test by NCSP participants. Our study showed that elderly participants preferred FIT, whereas participants with higher education and income levels as well as those with family members or acquaintances with CRC preferred colonoscopy. The current study is the first to evaluate primary CRC screening method preferences in NCSP participants.

Four large randomized controlled trials are currently evalu- 
Table 4. Respondents' Experiences with and Opinions about the NCSP

\begin{tabular}{llc}
\hline & \multicolumn{1}{c}{ Opinion } & No. (\%) \\
\hline Experience of NCSP participation & Annually-frequently & $357(90.2)$ \\
& Often-infrequently & $(9.8)$ \\
Experience of FIT participation & & $56(14.1)$ \\
Experience of FIT participation & None & $44(12.9)$ \\
Bad experience from FIT & Yes & $35(77.8)$ \\
Cause of bad experience from FIT & Stool sampling & $9(20.0)$ \\
& Stool storage and transportation & $1(2.2)$ \\
Experience of colonoscopy participation & Other & $134(33.8)$ \\
Experience of colonoscopy participation & & $103(39.3)$ \\
Bad experience with colonoscopy & None & $73(68.9)$ \\
Cause of bad experience from colonoscopy & Yes & Complicated preparation process \\
& Complicated colonoscopy process itself & $27(25.5)$ \\
\hline
\end{tabular}

NCSP, National Cancer Screening Program; FIT, fecal immunochemical test.

ating the completion rate of a primary colonoscopy screening program, ${ }^{24-27}$ and the long-term results are expected in the next decade. In a randomized controlled trial comparing primary colonoscopy with biannual FIT in Spain, the participation rate was higher in FIT group than in colonoscopy group (34.2\% vs 24.6\%, p<0.001). ${ }^{24}$ The different participation rates resulted in the same CRC detection rate in both groups. In a recent German population-based case-control study, ${ }^{28}$ only $1.7 \%$ of the patients with CRC underwent screening colonoscopy versus $12 \%$ of the patients without CRC. In a meta-analysis of prospective CRC screening studies, ${ }^{29}$ the overall participation rates for FIT and colonoscopy were $42 \%$ and $28 \%$, respectively. In the United States, participation rates were higher for FIT group than colonoscopy group (58.8\% vs 42.4\%, respectively, $\mathrm{p}<0.001) .{ }^{23}$ Our study result contrasted those of previous studies ${ }^{23,24,29,30}$ in that colonoscopy was preferred to FIT; however, our findings may be cautiously interpreted as socioeconomic status, the capacity to perform colonoscopy, and perceived awareness of CRC may differ among countries. In Korea, the excellent accessibility and low cost of colonoscopy, and the availability of experienced colonoscopists may have influenced our results of a higher preference for colonoscopy. However, sufficient information should be provided to participants about the advantages and disadvantages of FIT versus colonoscopy to enable their informed decisions, as their choice may change after education. For example, in a CRC screening from Hong Kong, ${ }^{19}$ patients initially chose colonoscopy over FIT (47.4\% vs 21.8\%, respectively); however, $27.1 \%$ of participants changed to FIT from colonoscopy and $8 \%$ changed to colonoscopy from FIT after the educational session. As a result, the final participation rate was higher in the FIT group than in the colonoscopy group (61\% vs 39\%, respec- tively).

Considering the lower participation rate of CRC screening than those for other cancers, ${ }^{7-9}$ strategies to increase participation rates are needed. Accordingly, the choice of screening modality may encourage individuals to participate, as we noted substantial variation in CRC screening method preference by age, family history of CRC, and socioeconomic status. Furthermore, a detailed information system, such as interviews, an online system, or leaflets should be provided to increase the participation rate through their informed decisions. Considering that only $12.9 \%$ of participants reported a previous bad experience with FIT and 39.3\% experienced a bad experience with colonoscopy, sufficient information about the advantages and disadvantages of FIT and colonoscopy may be essential to increasing CRC screening participation rates. In a multi-criteria decision analysis, inconvenience, safety, and testing frequency were most significantly associated with intention to attend. ${ }^{30}$

This study had some limitations. First is the possibility of selection bias derived from its single-center design. In addition, there is likely some sampling bias in the participants since the complete overall response rate was only $36.2 \%$ and that all respondents are thought to be interested in the NCSP. Second, our study was based on a questionnaire rather than actual data from administrative or clinical trials. As demonstrated in a CRC screening program of Hong Kong, ${ }^{19}$ a significant number of participants may change their initial opinion just before the actual CRC screening tests are performed. Therefore, an actual clinical trial on this issue is warranted in the future. However, this kind of study may incur large economic and resource burdens. Third, this survey tool was not verified for the reliability and validity. However, members of "Committee of Endoscopy 
Quality Improvement in the Korean Society of Gastrointestinal Endoscopy" checked this survey tool as we failed to find any verified survey tools on this issue. Finally, previous history of colon polyp was not investigated in this study. As patients with experience of polypectomy may be more aware of importance of colon polyp removal, it would be better if they were excluded from this study.

In conclusion, colonoscopy was preferred to FIT at a ratio of 2.2 to 1 in NCSP participants in Korea. The demonstrated colonoscopy preference should be considered in the creation of a primary colonoscopy screening strategy in Korea. However, actual clinical trial data about this issue are needed.

\section{CONFLICTS OF INTEREST}

No potential conflict of interest relevant to this article was reported.

\section{ACKNOWLEDGEMENTS}

This study was supported by a National Cancer Center grant (1660740-1).

Author contributions: Y.H.C., D.H.K., and J.M.C. contributed to the conception and design of the study and were responsible for the acquisition, analysis, and interpretation of data. Y.T.J., J.S.M., J.O.K., S.K.L., and Y.K.C. were responsible for the analysis and interpretation of data as well as writing a manuscript. J.P.I., J.Y.J., J.E.S., S.M.Y., Y.J., E.S.K., K.N.L., and S.J.C. revised the manuscript. Y.K. and B.Y.P. rechecked final manuscript and English revision. All authors read and approved the final manuscript.

\section{REFERENCES}

1. Lin OS, Kozarek RA, Cha JM. Impact of sigmoidoscopy and colonoscopy on colorectal cancer incidence and mortality: an evidence-based review of published prospective and retrospective studies. Intest Res 2014;12:268-274.

2. Schreuders EH, Ruco A, Rabeneck L, et al. Colorectal cancer screening: a global overview of existing programmes. Gut 2015;64:16371649.

3. van der Steen A, Knudsen AB, van Hees F, et al. Optimal colorectal cancer screening in states' low-income, uninsured populations: the case of South Carolina. Health Serv Res 2015;50:768-789.

4. Gupta S, Halm EA, Rockey DC, et al. Comparative effectiveness of fecal immunochemical test outreach, colonoscopy outreach, and usual care for boosting colorectal cancer screening among the underserved: a randomized clinical trial. JAMA Intern Med 2013;173:1725-1732.

5. Shin A, Choi KS, Jun JK, et al. Validity of fecal occult blood test in the national cancer screening program, Korea. PLoS One 2013;8:e79292.
6. Yoo KY. Cancer control activities in the Republic of Korea. Jpn J Clin Oncol 2008;38:327-333.

7. Vleugels JL, van Lanschot MC, Dekker E. Colorectal cancer screening by colonoscopy: putting it into perspective. Dig Endosc 2016;28:250-259.

8. Klabunde CN, Lanier D, Meissner HI, Breslau ES, Brown ML. Improving colorectal cancer screening through research in primary care settings. Med Care 2008;46(9 Suppl 1):S1-S4.

9. Meissner HI, Breen N, Klabunde CN, Vernon SW. Patterns of colorectal cancer screening uptake among men and women in the United States. Cancer Epidemiol Biomarkers Prev 2006;15:389394.

10. Almog R, Ezra G, Lavi I, Rennert G, Hagoel L. The public prefers fecal occult blood test over colonoscopy for colorectal cancer screening. Eur J Cancer Prev 2008;17:430-437.

11. Janz NK, Lakhani I, Vijan S, Hawley ST, Chung LK, Katz SJ. Determinants of colorectal cancer screening use, attempts, and nonuse. Prev Med 2007;44:452-458.

12. Ling BS, Moskowitz MA, Wachs D, Pearson B, Schroy PC. Attitudes toward colorectal cancer screening tests. J Gen Intern Med 2001;16:822-830.

13. Schroy PC 3rd, Lal S, Glick JT, Robinson PA, Zamor P, Heeren TC. Patient preferences for colorectal cancer screening: how does stool DNA testing fare? Am J Manag Care 2007;13:393-400.

14. Schroy PC 3rd, Emmons K, Peters E, et al. The impact of a novel computer-based decision aid on shared decision making for colorectal cancer screening: a randomized trial. Med Decis Making 2011;31:93-107.

15. Hawley ST, Volk RJ, Krishnamurthy P, Jibaja-Weiss M, Vernon SW, Kneuper S. Preferences for colorectal cancer screening among racially/ethnically diverse primary care patients. Med Care 2008;46(9 Suppl 1):S10-S16.

16. DeBourcy AC, Lichtenberger S, Felton S, Butterfield KT, Ahnen DJ, Denberg TD. Community-based preferences for stool cards versus colonoscopy in colorectal cancer screening. J Gen Intern Med 2008;23:169-174.

17. Wolf RL, Basch CE, Brouse CH, Shmukler C, Shea S. Patient preferences and adherence to colorectal cancer screening in an urban population. Am J Public Health 2006;96:809-811.

18. Pignone M, Bucholtz D, Harris R. Patient preferences for colon cancer screening. J Gen Intern Med 1999;14:432-437.

19. Wong MC, John GK, Hirai HW, et al. Changes in the choice of colorectal cancer screening tests in primary care settings from 7,845 prospectively collected surveys. Cancer Causes Control 2012;23:1541-1548.

20. Naing C, Jun YK, Yee WM, et al. Willingness to take a screening test for colorectal cancer: a community-based survey in Malaysia. Eur J Cancer Prev 2014;23:71-75.

21. Saengow U, Chongsuwiwatvong V, Geater A, Birch S. Preferences and acceptance of colorectal cancer screening in Thailand. Asian Pac J Cancer Prev 2015;16:2269-2276.

22. Wong MC, Ching JY, Chan VC, Sung JJ. The comparative cost- 
effectiveness of colorectal cancer screening using faecal immunochemical test vs. colonoscopy. Sci Rep 2015;5:13568.

23. Singal AG, Gupta S, Tiro JA, et al. Outreach invitations for FIT and colonoscopy improve colorectal cancer screening rates: a randomized controlled trial in a safety-net health system. Cancer 2016;122:456-463.

24. Quintero E, Castells A, Bujanda L, et al. Colonoscopy versus fecal immunochemical testing in colorectal-cancer screening. N Engl J Med 2012;366:697-706.

25. ClinicalTrials.gov. CONFIRM trial [Internet]. Bethesda: U.S. National Library of Medicine; c2016 [cited 2016 Dec 19]. Available from: http://www.clinicaltrials.gov/ct2/show/NCT01239082.

26. Kaminski MF, Bretthauer M, Zauber AG, et al. The NordICC study: rationale and design of a randomized trial on colonoscopy screening for colorectal cancer. Endoscopy 2012;44:695-702.
27. ClinicalTrials.gov. SCREESCO trial [Internet]. Bethesda: U.S. National Library of Medicine; c2016 [cited 2016 Dec 19]. Available from: http://www.clinicaltrials.gov/ct2/show/NCT02078804.

28. Brenner H, Chang-Claude J, Jansen L, Knebel P, Stock C, Hoffmeister M. Reduced risk of colorectal cancer up to 10 years after screening, surveillance, or diagnostic colonoscopy. Gastroenterology 2014;146:709-717.

29. Khalid-de Bakker C, Jonkers D, Smits K, Mesters I, Masclee A, Stockbrügger R. Participation in colorectal cancer screening trials after first-time invitation: a systematic review. Endoscopy 2011;43:1059-1086.

30. Hummel JM, Steuten LG, Groothuis-Oudshoorn CJ, Mulder N, Ijzerman MJ. Preferences for colorectal cancer screening techniques and intention to attend: a multi-criteria decision analysis. Appl Health Econ Health Policy 2013;11:499-507. 